\title{
MONITORAMENTO DE IRREGULARIDADES IONOSFERICAS DURANTE OS ANOS DE 2008-2010 E SEUS EFEITOS SOBRE OS SINAIS DE RADIO
}

\author{
Pâmela Rita Pereira Meibach Rosa ${ }^{1}$ \\ Marcio Tadeu Honorato de Assis Muella ${ }^{2}$
}

Resumo: As irregularidades ionosféricas que podem interferir em sinais de comunicação GPS estão fortemente relacionadas com a densidade do plasma ionosférico. A densidade da atmosfera na qual a ionosfera está inserida varia com a atividade solar, afetando com isso a sua distribuição vertical de ionização. Desta forma, durante o período de mínimo na atividade solar as irregularidades ionosféricas tendem a ser menos desenvolvidas. O período de mínimo solar entre os ciclos 23-24 foi um dos mais baixos e longos da história e ocorreu entre os anos de 2007-2009. Neste estudo é apresentada uma análise dos dados de monitoramento ionosférico obtidos entre 2008-2010 nas estações brasileiras de São José dos Campos (23, $\left.2^{\circ} \mathrm{S} ; 45,9^{\circ} \mathrm{W}\right)$ e Palmas $\left(10,2^{\circ} \mathrm{S} ; 48,2^{\circ} \mathrm{W}\right)$. O principal objetivo é investigar, através das suas assinaturas nos registros dos instrumentos de monitoramento, a atividade das irregularidades ionosféricas que ocorreram próximo ao último período de mínimo solar. A metodologia empregada possibilita estudar irregularidades com diferentes tamanhos de escala observadas a partir de vários instrumentos, tal como, imageadores all-sky, ionossonda e sinais de amplitude e fase do GPS. Importantes casos foram obtidos e analisados em virtude de suas características morfológicas e propriedades físicas relacionadas.

Palavras-chave: Irregularidade ionosférica; Bolhas de plasma; Mínimo solar.

\footnotetext{
1 Mestrado em Física e Astronomia/Instituto de Pesquisa e Desenvolvimento/UNIVAP, Brasil. E-mail: pamelaryta@gmail.com.

2 Mestrado em Física e Astronomia/Instituto de Pesquisa e Desenvolvimento/UNIVAP, Brasil. E-mail: mmuella@univap.br.
} 